\title{
Texture analysis of vertebral bone marrow using chemical shift encoding-based water-fat MRI: a feasibility study
}

\author{
E. Burian ${ }^{1}$ (D) $\cdot$ K. Subburaj ${ }^{2}$ - M. R. K. Mookiah ${ }^{2} \cdot$ A. Rohrmeier ${ }^{1}$ - D. M. Hedderich ${ }^{1} \cdot$ M. Dieckmeyer $^{1,3} \cdot$ M. N. Diefenbach ${ }^{3}$. \\ S. Ruschke ${ }^{3}$ - E. J. Rummeny ${ }^{3}$. C. Zimmer ${ }^{1}$ - J. S. Kirschke ${ }^{1}$ - D. C. Karampinos ${ }^{3} \cdot$ T. Baum $^{1}$
}

Received: 10 October 2018 / Accepted: 18 February 2019 / Published online: 22 March 2019

(C) The Author(s) 2019

\begin{abstract}
Summary This feasibility study investigated the spatial heterogeneity of the lumbar vertebral bone marrow using chemical shift encoding-based water-fat MRI. Acquired texture features like contrast and dissimilarity allowed for differentiation of pre- and postmenopausal women and may serve as imaging biomarkers in the future.

Introduction While the vertebral bone marrow fat using chemical shift encoding water-fat magnetic resonance imaging (MRI) has been extensively studied, its spatial heterogeneity has not been analyzed yet. Therefore, this feasibility study investigated the spatial heterogeneity of the lumbar vertebral bone marrow by using texture analysis in proton density fat fraction (PDFF) maps.

Methods Forty-one healthy pre- and postmenopausal women were recruited for this study (premenopausal $(n=15) 30 \pm 7$ years, postmenopausal $(n=26) 65 \pm 7$ years). An eight-echo 3D spoiled gradient echo sequence was used for chemical shift encodingbased water-fat separation at the lumbar spine. Vertebral bodies L1 to L5 were manually segmented. Mean PDFF values and texture features were extracted at each vertebral level, namely variance, skewness, and kurtosis, using statistical moments and second-order features (energy, contrast, correlation, homogeneity, dissimilarity, entropy, variance, and sum average). Parameters were compared between pre- and postmenopausal women and vertebral levels.

Results PDFF was significantly higher in post- than in premenopausal women $(49.37 \pm 8.14 \%$ versus $27.76 \pm 7.30 \%, p<0.05)$. Furthermore, PDFF increased from L1 to L5 (L1 37.93 $\pm 12.85 \%$, L2 38.81 $\pm 12.77 \%$, L3 40.23 $\pm 12.72 \%$, L4 42.80 $\pm 13.27 \%$, L5 $45.21 \pm 14.55 \%, p<0.05)$. Bone marrow heterogeneity based on texture analysis was significantly $(p<0.05)$ increased in

\author{
E. Burian \\ e.burian@gmx.net \\ K. Subburaj \\ subburaj@sutd.edu.sg \\ M. R. K. Mookiah \\ mrk2k2@gmail.com \\ A. Rohrmeier \\ alexander.Rohrmeier@t-online.de \\ D. M. Hedderich \\ dennis.hedderich@tum.de \\ M. Dieckmeyer \\ michael.dieckmeyer@tum.de \\ M. N. Diefenbach \\ maximilian.diefenbach@tum.de \\ S. Ruschke \\ stefan.ruschke@tum.de \\ E. J. Rummeny \\ ernst.rummeny@tum.de
}

\author{
C. Zimmer \\ J. S. Kirschke \\ D. C. Karampinos \\ dimitrios.karampinos@tum.de \\ T. Baum \\ thomas.baum@tum.de
}

claus.zimmer@tum.de

jan.kirschke@tum.de

Department of Diagnostic and Interventional Neuroradiology, Klinikum rechts der Isar, Technical University of Munich, Ismaninger Str. 22, 81675 Munich, Germany

2 Pillar of Engineering Product Development, Singapore University of Technology and Design, 8 Somapah Road, Singapore 487372, Singapore

3 Department of Diagnostic and Interventional Radiology, Klinikum rechts der Isar, Technical University of Munich, Ismaninger Str. 22, 81675 Munich, Germany
\end{abstract}


postmenopausal women. Contrast and dissimilarity performed best in differentiating pre- and postmenopausal women (AUC $=0.97$ and 0.96, respectively), not significantly different compared with PDFF (AUC $=0.97)$.

Conclusion Conclusively, an increased bone marrow heterogeneity could be observed in postmenopausal women. In the future, texture parameters might provide additional information to detect and monitor vertebral bone marrow alterations due to aging or hormonal changes beyond conventional anatomic imaging.

Keywords Bone marrow $\cdot$ Magnetic resonance imaging $\cdot$ Osteoporosis $\cdot$ Spine $\cdot$ Texture

\section{Introduction}

The non-mineralized part of the bone consists of the red and yellow bone marrow, which cover endocrine and hematopoietic functions and differ in respects of composition and vascularization $[1,2]$. The quantitative composition of bone marrow has been assessed using single-voxel proton magnetic resonance spectroscopy (MRS) and chemical shift encoding-based water-fat magnetic resonance imaging (MRI). This allows for calculating surrogate parameters like proton density fat fraction (PDFF) and with MRS the identification of the chemical structure of fatty acids and their magnitude [3-5].

In aging and due to pathophysiological changes associated with endocrine or metabolic diseases like osteoporosis and type 2 diabetes, vertebral bone marrow alterations with shifts towards greater bone marrow adiposity and lower unsaturation levels were observed [6-8]. Decreasing bone mineral density (BMD) clinically represents an issue which can be detected in a characteristic distribution pattern in the elderly and people suffering from osteoporosis but also due to neoplastic diseases [9]. To evaluate the fracture risk associated with bone mineral loss, Dual Energy XAbsorptiometry (DXA) poses the preferred technique in a clinical setting. However, this method has inherent limitations. DXA is prone to confounding effects like local excessive fat tissue deposition and by inadequate assessment of local density inhomogeneities [10,11]. Moreover, it has been shown that a high percentage of vertebral fractures occur in individuals not classified as osteoporotic or osteopenic according to DXA measurements [12]. MRI offers the possibility to investigate on spatial changes in bone marrow composition by detecting fat content and depicting tissue structure quantitatively $[13,14]$. Thus, it enables the investigator to calculate subtle changes in vertebral bone marrow composition and to stratify the potential fracture risk [13].

In the past, PDFF has been shown to be a valid biomarker for evaluating fatty infiltration of vertebral bone marrow with an increase from the cervical to the lumbar spine in children and adults [4, 15]. Baum et al. [16] reported an accelerated fatty conversion of the vertebral bone marrow in females compared with males with increasing age particularly evident after menopause. Ruschke et al. [15] showed that detectable changes of vertebral fat marrow content and in this way an increase of PDFF not only occur in the adult vertebral column, but also can likewise be visualized during childhood. Relative age-related PDFF changes showed an anatomical variation with the most pronounced changes at lower lumbar vertebral levels in both sexes [4]. Furthermore, PDFF shows specific distribution patterns inter- and intraindividually dependent on hormonal changes (e.g., induced by menopause) and on the topography $[3,13,17]$.

To detect structural changes qualitatively in terms of signal loss appearing in age- or malignancy-associated bone marrow alterations in the clinical routine, conventional anatomic imaging using T1- or T2-weighed sequences have been implemented in standard examination protocols $[18,19]$. More advanced methods which allow for a quantitative evaluation like PDFF calculation are on the edge of being transferred into clinical use, because of the rising evidence for serving as a valid biomarker for muscular and skeletal fat accumulation $[3$, $13,14,20]$. Still, the heterogeneity of the vertebral bone marrow using texture analysis based on chemical shift encoding water-fat MRI has not been analyzed.

The texture of an image can be defined as spatial arrangement of pixels with different intensities [21]. Texture measures can quantify the gray level variations reflecting repetitive patterns and uniformity in the image pixels, e.g., by using gray Haralick's level co-occurrence matrix (GLCM) [22]. These texture parameters have been used for trabecular bone microstructure analysis in computed tomography (CT) scans and can similarly be applied on MR-based PDFF maps [21]. Additionally, certain texture features were shown to be of diagnostic help in identifying soft tissue malignancies from mammograms or fracture risk in CT $[23,24]$. With that said, the methodic deduction of the concept of using texture parameters to attain a diagnostic gain and subsequent transfer to MRI as radiation-free modality is a logical step and of high clinical significance with implementation in risk stratification for potential fractures or tumor relapse.

The purpose of this feasibility study was to investigate the spatial heterogeneity of the lumbar vertebral bone marrow by using texture analysis in PDFF maps derived from chemical shift encoding-based water-fat MRI in pre- and postmenopausal women and to compare the performance of the different parameters in differentiating the two groups. 


\section{Materials and methods}

\section{Subjects}

The study was approved by the local institutional committee for human research. All subjects gave written informed consent before participation in the study.

Healthy pre- and postmenopausal women were included in this study. Exclusion criteria were history of pathological bone changes such as hematological or metabolic bone disorders aside from osteoporosis, history of diabetes, and contraindications for MR imaging. In total, 15 pre- and 26 postmenopausal women were recruited. Subjects included in this study received no antiresorptive medication like bisphosphonates and denosumab. According to medically indicated DXA measurements, 17 postmenopausal women had normal BMD values, five were in the osteopenic range, and four were classified as osteoporotic.

\section{MR imaging}

All subjects underwent 3 T MRI (Ingenia, Philips Healthcare, Best, The Netherlands). An eight-echo 3D spoiled gradient echo sequence was used for chemical shift encoding-based water-fat separation at the lumbar spine using the built-in-the-table posterior coil elements (12channel array). The sequence acquired the eight echoes in a single TR using non-flyback (bipolar) readout gradients and the following imaging parameters: TR/TE $1 / \Delta \mathrm{TE}=11 /$ $1.4 / 1.1 \mathrm{~ms}, \mathrm{FOV}=220 \times 220 \times 80 \mathrm{~mm}^{3}$, acquisition matrix size $=124 \times 121$, acquisition voxel size $=1.8 \times 1.8 \times$ $4.0 \mathrm{~mm}^{3}$, receiver bandwidth $=1527 \mathrm{~Hz} /$ pixel, frequency direction $=\mathrm{A} / \mathrm{P}$ (to minimize breathing artifacts), 1 average, and scan time $=1 \mathrm{~min}$ and $17 \mathrm{~s}$. A flip angle of $3^{\circ}$ was used to minimize T1-bias effects.

\section{Vertebral bone marrow fat quantification}

The gradient echo imaging data were processed online using the fat quantification routine of the MR vendor. The routine procedure first performs a phase error correction and then a complex-based water-fat decomposition using a pre-calibrated seven-peak fat spectrum and a single $\mathrm{T}_{2} *$ to model the signal variation with echo time. The imaging-based proton density fat fraction (PDFF) map was computed as the ratio of the fat signal over the sum of fat and water signals. The vertebral bodies L1 to L 5 were included in the analysis and manually segmented by a radiologist (Fig. 1). The posterior elements and sclerotic changes of the endplates were excluded. Segmentation was performed on the PDFF maps by using the free open-source software Medical Imaging Interaction Toolkit (MITK, developed by the Division of Medical and

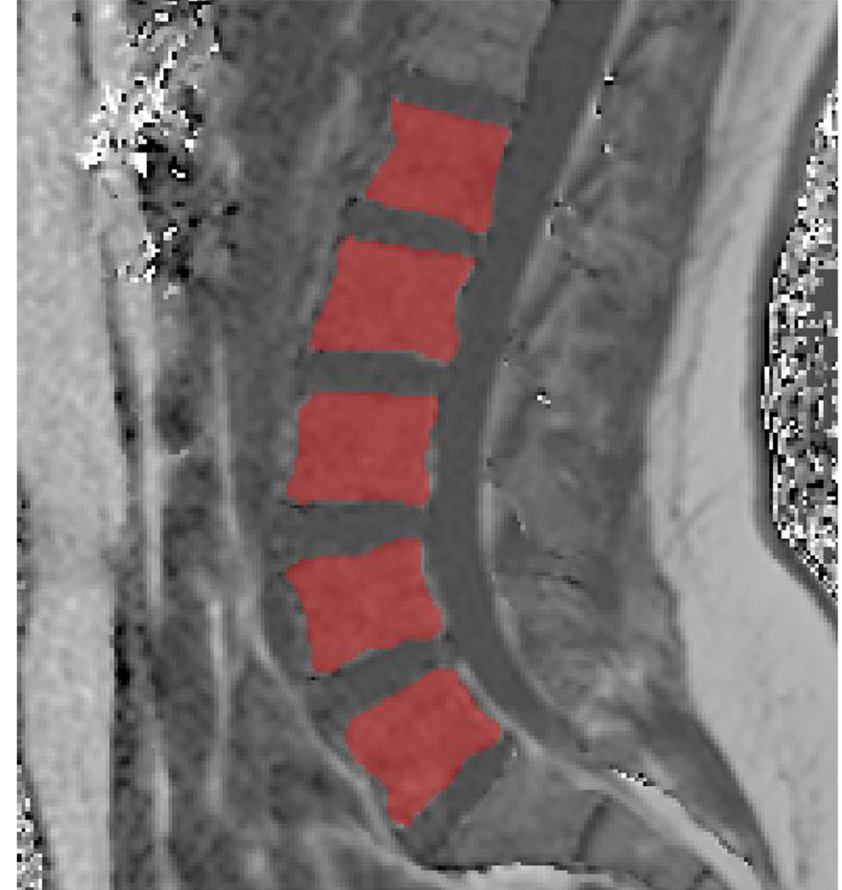

Fig. 1 Representative segmentation of lumbar vertebral bodies 1 to 5 in the PDFF map of a 22 year old woman

Biological Informatics, German Cancer Research Center, Heidelberg, Germany; www.mitk.org).

\section{Texture analysis}

Texture analysis was performed on vertebral bodies (L1 to L5) using GLCM [13]. Initially, gray level quantization was performed to prevent sparseness by normalizing the image intensities using the maximum gray level present in an image. The GCLM metrics were obtained from 16-bit images [25-27]. The statistical moments (variance, skewness, and kurtosis) and second-order GLCM features (energy, entropy, contrast, homogeneity, correlation, sum average, variance, and dissimilarity) were determined. The features quantify smoothness, roughness, and heterogeneity in an image. GLCM computes the joint probability of two adjacent voxel intensities at a given offset $d=(d x, d y, d z)$ and angular directions $\theta=\left(0^{\circ}, 45^{\circ}, 90^{\circ}\right.$, and $\left.135^{\circ}\right)[21,22]$. Where $d x$ and $d y$ denotes the displacement along $x$ and $y$ axis; $d z$ denotes the displacement along $z$ axis to compute the cooccurrence of voxel intensities at a given offset $d$ and a specific angular direction $\theta$ [28]. The co-occurrence probabilities of voxel intensities were computed from 26 neighbors, aligned in 13 directions. The mean value of the features computed from the 13 directions ensures the rotation invariance [28]. The gray level uniform quantization and texture analysis were performed using MATLAB 2017 (MathWorks Inc., Natick, MA, USA). 


\section{Statistical analysis}

The statistical analyses were performed with SPSS (SPSS Inc., Chicago, IL, USA). All tests were done using a twosided 0.05 level of significance.

The Kolmogorov-Smirnov test indicated no normally distributed data for the majority of parameters. Mean and standard deviation (SD) of PDFF and texture parameters averaged over L1 to L5 were computed for pre- and postmenopausal women and compared using the Mann-Whitney tests. Furthermore, receiver operating characteristics (ROC) were performed to assess the performance of PDFF and texture parameters averaged over L1 to L5 to differentiate pre- and postmenopausal women and reported as area under the curve (AUC) values. AUC values were comparted by the method proposed by Hanley and Mc Neil [29]. The Friedmann tests for PDFF/texture parameters and entering L1 to L5 as group variable were preformed testing whether any significant differences between all five vertebral levels exist. This was done in pre- and postmenopausal women separately.

The Spearman correlations coefficients $r$ were computed to investigate the association of PDFF and texture parameters with age and BMI. Linear regression models were used to adjust the differences in textural parameters between preand postmenopausal women for mean PDFF and age.

\section{Results}

\section{Study population}

Our study population consisted of 15 premenopausal and 26 postmenopausal women. Subjects in the premenopausal group were aged $30 \pm 7$ years and those in the postmenopausal group $65 \pm 7$ years. Both groups did not significantly $(p>0.05)$ differ in BMI (Table 1).

\section{PDFF measurements}

Mean PDFF values averaged over L1 to L5 showed statistically significant differences between pre- to postmenopausal women $(27.76 \pm 7.31 \%$ versus $49.37 \pm 8.14 \% ; p<0.001$; AUC $=0.97$; Table 1). PDFF significantly $(p<0.001)$ increased from L1 to L5 in both groups (Table 2).

\section{Texture analysis}

Eleven texture features were computed in pre- and postmenopausal women as shown in Table 1. Contrast and dissimilarity differentiated the pre- and postmenopausal best with AUC of 0.97 and 0.96 , respectively (Fig. 2). Kurtosis global, correlation, and sum average showed no significant difference between pre- and postmenopausal women $(p>0.05)$. In contrast to
PDFF, all texture features except for correlation showed no statistically significant anatomical variations from L1 to L5 $(p>0.05$; Table 2). However, correlation showed no consistent trend from L1 to L5 (Table 2). No significant $(p>0.05)$ differences between the AUCs based on mean PDFF values and AUCs based on texture features contrast and dissimilarity could be detected.

\section{Correlations}

Significant correlations were detected between age and PDFF $(r=0.703, p<0.0001)$, contrast $(r=0.626, p<0.0001)$, and dissimilarity $(r=0.470, p<0.0001)$, respectively (Table 3 and Fig. 3). BMI showed no significant correlations with age, PDFF, and texture parameters $(p>0.05$; Table 3 and Fig. 3).

\section{Linear regression}

Adjusting for PDFF as a control variable, contrast, $(p=0.011)$ and dissimilarity $(p=0.009)$ showed significant differences between pre- and postmenopausal women. Adjusting for age resulted in no significant differences in PDFF, dissimilarity, and contrast between the two groups $(p>0.05)$.

\section{Discussion}

This study demonstrated that postmenopausal women had not only an increased lumbar vertebral bone marrow PDFF, but also a greater bone marrow heterogeneity as assessed by texture analysis in PDFF maps compared with premenopausal women.

Texture analysis was firstly described by Haralick in the 1970s as a tool for classification of imaging features in general like photographic or satellite images, introducing 28 parameters like contrast, correlation, and entropy among others [22]. Since then, a large and increasing number of researchers have used these texture features for medical imaging analysis, e.g., in CT, MRI, FDG-PET, and ultrasound [28, 30-32]. Besides its recent multifold use in oncological imaging in terms of tissue entity discrimination, characterization, and treatment response monitoring, texture analysis also was described as a reproducible tool to quantitatively assess paraspinal fatty infiltration in MRI [33-36]. In these and other studies, texture heterogeneity was described to be associated with therapy response and clinical outcome [35]. Besides MRI, the use of texture analysis was investigated on using mammography and in $\mathrm{CT}$ in the past to analyze its capability to contribute to computer-aided cancer diagnosis or bone quality measurements [23, 24].

In this feasibility study, we showed that bone marrow heterogeneity, analogously to PDFF, increases significantly 
Table 1 Subject characteristics (age and BMI), PDFF values, and texture features averaged over L1 to L5 in pre- and postmenopausal women. Parameters were compared between the two groups with the Mann-Whitney tests ( $p$ values) and receiver operator characteristics (area under curve (AUC)). Status: 0 premenopausal, 1 postmenopausal

\begin{tabular}{|c|c|c|c|c|c|c|}
\hline & Status & $n$ & Mean & $\mathrm{SD}$ & AUC & $p$ \\
\hline \multirow[t]{2}{*}{ Age } & 0 & 15 & 30 & 7 & & \multirow[t]{2}{*}{$<0.001$} \\
\hline & 1 & 26 & 65 & 7 & & \\
\hline \multirow[t]{2}{*}{ BMI } & 0 & 15 & 25.8 & 1.4 & & \multirow[t]{2}{*}{0.464} \\
\hline & 1 & 26 & 25.4 & 4.3 & & \\
\hline \multirow[t]{2}{*}{ PDFF (L1-5) } & 0 & 15 & 27.76 & 7.31 & \multirow[t]{2}{*}{0.97} & \multirow[t]{2}{*}{$<0.001$} \\
\hline & 1 & 26 & 49.37 & 8.14 & & \\
\hline \multirow[t]{2}{*}{ Variance $_{\text {global }}$} & 0 & 15 & 17.97 & 1.97 & \multirow[t]{2}{*}{0.71} & \multirow[t]{2}{*}{0.042} \\
\hline & 1 & 26 & 19.49 & 2.53 & & \\
\hline \multirow[t]{2}{*}{ Skewness global } & 0 & 15 & 2.18 & 0.25 & \multirow[t]{2}{*}{0.30} & \multirow[t]{2}{*}{0.032} \\
\hline & 1 & 26 & 1.99 & 0.25 & & \\
\hline \multirow[t]{2}{*}{ Kurtosis $_{\text {global }}$} & 0 & 15 & 2.92 & 1.15 & \multirow[t]{2}{*}{0.32} & \multirow[t]{2}{*}{0.062} \\
\hline & 1 & 26 & 2.186 & 1.015 & & \\
\hline \multirow[t]{2}{*}{ Energy } & 0 & 15 & 0.694 & 0.039 & \multirow[t]{2}{*}{0.26} & \multirow[t]{2}{*}{0.012} \\
\hline & 1 & 26 & 0.657 & 0.043 & & \\
\hline \multirow[t]{2}{*}{ Contrast } & 0 & 15 & 116,340 & 16,788 & \multirow[t]{2}{*}{0.97} & \multirow[t]{2}{*}{$<0.001$} \\
\hline & 1 & 26 & 172,652 & 23,811 & & \\
\hline \multirow[t]{2}{*}{ Entropy } & 0 & 15 & 3.227 & 0.443 & \multirow[t]{2}{*}{0.79} & \multirow[t]{2}{*}{0.002} \\
\hline & 1 & 26 & 3.750 & 0.505 & & \\
\hline \multirow[t]{2}{*}{ Homogeneity } & 0 & 15 & 0.838 & 0.023 & \multirow[t]{2}{*}{0.24} & \multirow[t]{2}{*}{0.007} \\
\hline & 1 & 26 & 0.815 & 0.026 & & \\
\hline \multirow[t]{2}{*}{ Correlation } & 0 & 15 & 0.845 & 0.010 & \multirow[t]{2}{*}{0.60} & \multirow[t]{2}{*}{0.317} \\
\hline & 1 & 26 & 0.847 & 0.010 & & \\
\hline Sum $_{\text {average }}$ & 0 & 15 & 0.000046 & 0.000006 & 0.44 & 0.534 \\
\hline & 1 & 26 & 0.000043 & 0.000012 & & \\
\hline Variance & 0 & 15 & 0.068 & 0.008 & 0.71 & 0.026 \\
\hline & 1 & 26 & 0.075 & 0.010 & & \\
\hline Dissimilarity & 0 & 15 & 77.48 & 9.65 & 0.96 & $<0.001$ \\
\hline & 1 & 26 & 101.91 & 12.18 & & \\
\hline
\end{tabular}

in postmenopausal women. In contrast to increasing PDFF from L1-5 however, bone marrow heterogeneity remained constant from L1 to L5. One possible hypothesis for this finding from a pathophysiologic point of view might be due to the transformation pattern from red to yellow bone marrow starting from solitary foci $[1,37]$. The temporal discrepancy regarding the starting point of vertebral bone marrow fatty conversion beginning from L5 and visualized by increasing PDFF values from the cervical to the lumbar spine has been reported previously [15]. The results of texture analysis presented in this study imply a rather homogenous spatial fatty bone marrow conversion at different vertebral levels despite the differing time of the initial onset the structural changes are taking place. Although experimental preclinical studies have been conducted repetitively proving the negative correlation of increasing bone marrow fat and trabecular structure in animal models, the spatial replacement pattern in humans still remains unclear in this study giving us a hint towards anatomical homogeneous bone marrow changes [1, 38].
Dissimilarity and contrast outperformed the other Haralick texture features calculated from GLCM and showed comparable discrimination power to PDFF in differentiating between pre- and postmenopausal women (AUC $=0.97$ for contrast, $\mathrm{AUC}=0.96$ for dissimilarity, and $\mathrm{AUC}=0.97$ for PDFF, respectively). Neither the AUC values for dissimilarity and PDFF nor the AUC values for contrast and PDFF showed significant differences regarding the differentiation of the two groups.

To our knowledge, this study is the first to verify an increased bone marrow heterogeneity in postmenopausal women by use of texture analysis. Structural musculoskeletal changes like increasing bone marrow adiposity due to aging, hormonal changes, and endocrine or metabolic diseases have been described extensively in the past [5, 6]. Baum et al. [3, 4] visualized these changes in the lumbar spine using chemical shift encoding-based water-fat MRI and showed an increasing bone marrow adiposity from L1 to L5 as well as in postmenopausal period. Texture parameters (except correlation) showed no significant difference concerning anatomical 
Table 2 PDFF values and texture features in L1 to L5 in pre- and postmenopausal women. Differences between vertebral levels L1 to L5 were evaluated using the Friedmann tests in pre- and postmenopausal women, separately

\begin{tabular}{|c|c|c|c|c|c|c|}
\hline & \multicolumn{3}{|c|}{ Premenopausal women } & \multicolumn{3}{|c|}{ Postmenopausal women } \\
\hline & Mean & $\mathrm{SD}$ & $p$ value & Mean & $\mathrm{SD}$ & $p$ value \\
\hline L1 PDFF & 25.20 & 6.71 & & 45.58 & 8.94 & \\
\hline L2 PDFF & 26.43 & 6.54 & & 46.25 & 9.30 & \\
\hline L3 PDFF & 27.71 & 7.71 & $<0.001$ & 47.74 & 8.45 & $<0.001$ \\
\hline L4 PDFF & 29.14 & 7.77 & & 50.99 & 8.03 & \\
\hline L5 PDFF & 30.32 & 8.11 & & 54.14 & 9.16 & \\
\hline $\mathrm{L} 1$ variance $_{\text {global }}$ & 18.07 & 4.89 & & 17.43 & 5.57 & \\
\hline L2 variance $_{\text {global }}$ & 17.33 & 2.51 & & 19.04 & 3.97 & \\
\hline L3 variance global & 17.54 & 0.86 & 0.765 & 20.60 & 3.89 & 0.319 \\
\hline L4 variance global & 17.70 & 1.35 & & 18.97 & 2.46 & \\
\hline L5 variance global & 17.91 & 3.73 & & 17.71 & 4.93 & \\
\hline L1 skewness global & 2.33 & 0.47 & & 2.16 & 0.44 & \\
\hline L2 skewness global & 2.21 & 0.38 & & 2.07 & 0.35 & \\
\hline L3 skewness global & 2.29 & 0.21 & 0.837 & 1.89 & 0.25 & 0.112 \\
\hline L4 skewness global & 2.14 & 0.29 & & 2.09 & 0.31 & \\
\hline L5 skewness global & 2.22 & 0.44 & & 2.13 & 0.46 & \\
\hline L1 kurtosis global & 3.68 & 2.30 & & 2.93 & 1.93 & \\
\hline L2 kurtosis global & 3.05 & 1.70 & & 2.50 & 1.44 & \\
\hline L3 kurtosis ${ }_{\text {global }}$ & 3.33 & 0.96 & 0.823 & 1.70 & 0.93 & 0.128 \\
\hline L4 kurtosis global & 2.72 & 1.17 & & 2.57 & 1.23 & \\
\hline L5 kurtosis global & 3.16 & 2.09 & & 2.85 & 1.84 & \\
\hline L1 energy & 0.713 & 0.065 & & 0.683 & 0.074 & \\
\hline L2 energy & 0.699 & 0.061 & & 0.672 & 0.062 & \\
\hline L3 energy & 0.716 & 0.031 & 0.837 & 0.643 & 0.052 & 0.188 \\
\hline L4 energy & 0.691 & 0.051 & & 0.678 & 0.055 & \\
\hline L5 energy & 0.697 & 0.067 & & 0.678 & 0.084 & \\
\hline L1 contrast & 110,669 & 26,399 & & 164,307 & 42,933 & \\
\hline L2 contrast & 110,146 & 23,269 & & 157,725 & 29,226 & \\
\hline L3 contrast & 102,093 & 8253 & 0.374 & 171,344 & 40,098 & 0.349 \\
\hline L4 contrast & 115,915 & 21,989 & & 159,131 & 25,425 & \\
\hline L5 contrast & 128,277 & 28,704 & & 187,539 & 49,912 & \\
\hline L1 entropy & 2.984 & 0.670 & & 3.397 & 0.809 & \\
\hline L2 entropy & 3.167 & 0.654 & & 3.556 & 0.686 & \\
\hline L3 entropy & 3.000 & 0.349 & 0.736 & 3.901 & 0.608 & 0.099 \\
\hline L4 entropy & 3.259 & 0.541 & & 3.539 & 0.659 & \\
\hline L5 entropy & 3.146 & 0.694 & & 3.520 & 0.988 & \\
\hline L1 homogeneity & 0.849 & 0.037 & & 0.830 & 0.044 & \\
\hline L2 homogeneity & 0.841 & 0.036 & & 0.824 & 0.037 & \\
\hline L3 homogeneity & 0.851 & 0.018 & 0.765 & 0.807 & 0.032 & 0.188 \\
\hline L4 homogeneity & 0.837 & 0.030 & & 0.828 & 0.033 & \\
\hline L5 homogeneity & 0.840 & 0.038 & & 0.827 & 0.051 & \\
\hline L1 correlation & 0.833 & 0.014 & & 0.837 & 0.011 & \\
\hline L2 correlation & 0.847 & 0.010 & & 0.848 & 0.012 & \\
\hline L3 correlation & 0.852 & 0.010 & $<0.001$ & 0.851 & 0.026 & $<0.001$ \\
\hline L4 correlation & 0.848 & 0.013 & & 0.856 & 0.009 & \\
\hline L5 correlation & 0.830 & 0.013 & & 0.839 & 0.010 & \\
\hline L1 sum $_{\text {average }}$ & 0.000048 & 0.000016 & & 0.000040 & 0.000014 & \\
\hline L2 sum average & 0.000045 & 0.000010 & & 0.000044 & 0.000012 & \\
\hline L3 sum average & 0.000043 & 0.000004 & 0.878 & 0.000048 & 0.000012 & 0.057 \\
\hline L4 sum average & 0.000045 & 0.000006 & & 0.000041 & 0.000007 & \\
\hline L5 sum average & 0.000046 & 0.000014 & & 0.000038 & 0.000012 & \\
\hline L1 variance & 0.069 & 0.021 & & 0.068 & 0.022 & \\
\hline L2 variance & 0.066 & 0.012 & & 0.073 & 0.016 & \\
\hline L3 variance & 0.064 & 0.005 & 0.765 & 0.080 & 0.015 & 0.231 \\
\hline L4 variance & 0.067 & 0.007 & & 0.071 & 0.010 & \\
\hline L5 variance & 0.069 & 0.016 & & 0.069 & 0.019 & \\
\hline L1 dissimilarity & 74.30 & 17.48 & & 96.93 & 24.33 & \\
\hline L2 dissimilarity & 74.75 & 15.50 & & 96.30 & 18.55 & \\
\hline L3 dissimilarity & 69.70 & 5.47 & 0.780 & 104.07 & 20.95 & 0.493 \\
\hline L4 dissimilarity & 77.24 & 14.16 & & 93.57 & 15.26 & \\
\hline L5 dissimilarity & 81.60 & 18.44 & & 103.27 & 26.67 & \\
\hline
\end{tabular}


a

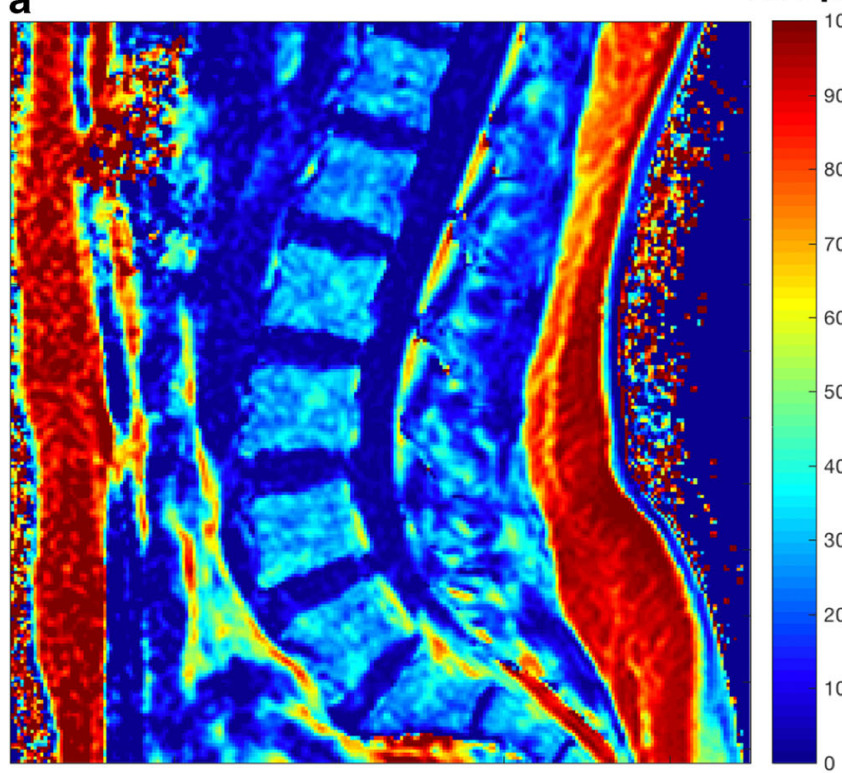

PDFF [\%]

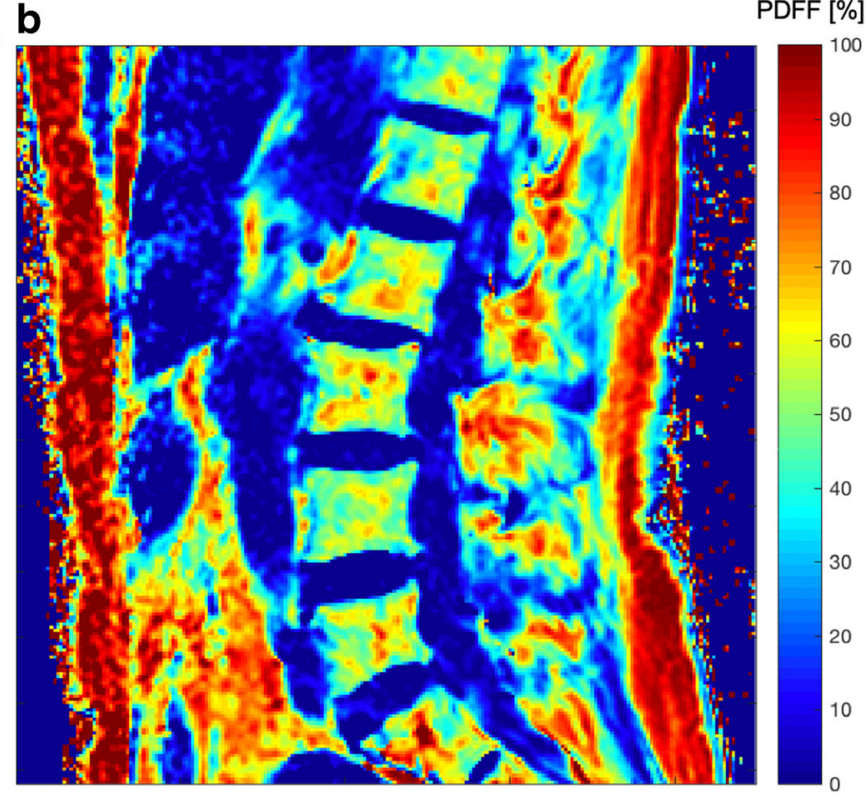

Fig. 2 Representative color-coded PDFF maps of the lumbar vertebral bone marrow of a premenopausal woman (age 22 years; mean PDFF 26.6\%; contrast 125,518, dissimilarity 82.97) (a) and a postmenopausal woman (age 71 years; mean PDFF 42.7\%; contrast 183,113, dissimilarity 108.75) (b)

variation through all scanned subjects. However, besides dissimilarity and contrast, other texture metrics like skewness, kurtosis, energy, and entropy proved to be risen after menopause with the latter showing the best result in differentiating post- from premenopausal women $(\mathrm{AUC}=0.79)$. With that said, it stands to reason that texture analysis for water-fat MRI may allow for similar diagnostic capabilities in the clinic, with additional benefit of being radiation free.

The texture feature "contrast" gives elements with similar gray level values a low weight. Elements with differing gray levels are given a high figure [22]. Texture "dissimilarity" is evaluated with the Kullback-Leibler divergence and can roughly be described as a measure of how different gray levels of two elements appear. "Entropy" accounts for a measure of randomness in pixel distribution and may depict clinically relevant changes in vertebral micro-architectural alterations. Other groups also investigated on the reliability of different texture parameters and proved that features like kurtosis, skewness, and uniformity showed good results in diagnostic and monitoring quality in cancer imaging [34, 35]. The described arbitrarily detected texture features are inherently dependent on imaging properties like resolution, noise, and scan parameters (repetition time, echo time, and receiver bandwidth) [39]. To ensure comparable signal-to-noise ratios throughout the scans, similar scan parameters and MRI protocols therefore should be used.

There are several limitations to this conducted study. First, to start with the methodical aspects confining the presented work, only 11 texture features were investigated on. Further parameters which showed good and reproducible results in other studies, e.g., uniformity, could be added to texture feature pool. Second, the heterogeneous distribution of healthy, osteopenic, and osteoporotic classified women can be mentioned. In a following study, postmenopausal women
Table 3 Correlation between subject characteristics (age and $\mathrm{BMI})$, PDFF values, and texture parameters (contrast and dissimilarity) in pre- and postmenopausal women. Parameters were compared with Spearman's rho test

\begin{tabular}{lllllll}
\hline & & Age & BMI & PDFF & Contrast & Dissimilarity \\
\hline Age & Spearman's rho & 1 & & 0.703 & 0.626 & 0.470 \\
{$[$ years $]$} & $p$ & - & n.s. & $<0.0001$ & $<0.0001$ & $<0.0001$ \\
BMI & Spearman's rho & & 1 & & & \\
{$\left[\mathrm{~kg} / \mathrm{m}^{2}\right]$} & $p$ & n.s. & - & n.s. & n.s. & n.s. \\
PDFF & Spearman's rho & 0.703 & & 1 & 0.796 & 0.599 \\
{$[\%]$} & $p$ & $<0.0001$ & n.s. & - & $<0.0001$ & $<0.0001$ \\
Contrast & Spearman's rho & 0.626 & & 0.796 & 1 & 0.948 \\
& $p$ & $<0.0001$ & n.s. & $<0.0001$ & - & $<0.0001$ \\
Dissimilarity & Spearman's rho & 0.470 & & 0.599 & 0.948 & 1 \\
& $p$ & $<0.0001$ & n.s. & $<0.0001$ & $<0.0001$ & - \\
\hline
\end{tabular}



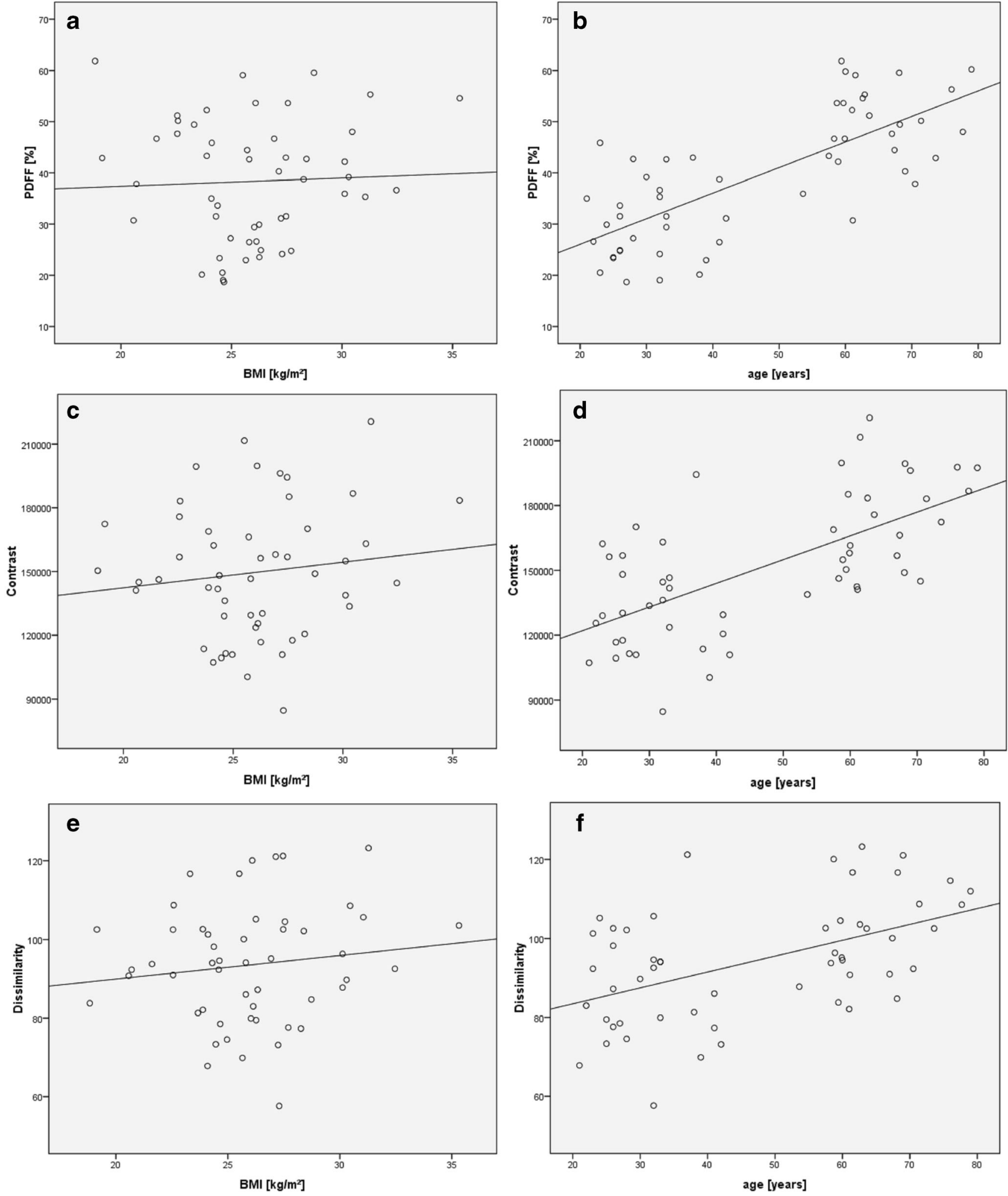

Fig. 3 PDFF, contrast, and dissimilarity are plotted against BMI (a, c, e) and age (b, d, f). PDFF, contrast, and dissimilarity correlate significantly with age

homogenously distributed into the three mentioned subgroups according to DXA measurements could be scanned and textural features within and across the groups could be adequately compared. This would be an important issue in diagnostic imaging and a step towards acquiring benchmark and threshold values for disease entity differentiation in MRI. 
In a further step, other diseases proven to be associated with structural changes in vertebral bone marrow composition and increasing PDFF values like type 2 diabetes mellitus could be in the focus of attention following the hints the presented study gave us concerning the connection between texture features, morphology, and pathophysiology $[3,14,16]$.

In conclusion, this study shows that texture features namely dissimilarity and contrast acquired by chemical shift encoding-based water-fat MRI can be used to describe spatial heterogeneity of vertebral bone marrow in pre- and postmenopausal women. These parameters might offer additional insight into vertebral bone marrow alterations due to aging or hormonal changes compared with established parameters like PDFF and enlighten osseous pathologic processes.

Funding The present work was supported by the European Research Council (grant agreement No 677661 - ProFatMRI and grant agreement No 637164 - iBack), TUM Faculty of Medicine KKF grant H01, and Philips Healthcare.

\section{Compliance with ethical standards}

\section{Conflicts of interest None.}

Ethical approval All procedures performed in this study using human participants were in accordance with the ethical standards with the institutional and the 1964 Helsinki declaration and its later amendments.

Abbreviations A/P, anterior/posterior; AUC, area under the curve; $\mathrm{BMD}$, bone mineral density; CT, computed tomography; DXA, Dualenergy X-ray absorptiometry; FDG-PET, fludeoxyglucose positron emission tomography; FOV, field of view; GLCM, gray Haralick's level cooccurrence matrix; L/R, left/right; L1, L2 etc., lumbar vertebrae 1, lumbar vertebrae 2 etc.; MRI, magnetic resonance imaging; MRS, single-voxel proton magnetic resonance spectroscopy; PDFF, proton density fat fraction; TR, repetition time; TE, echo time.

Open Access This article is distributed under the terms of the Creative Commons Attribution-NonCommercial 4.0 International License (http:// creativecommons.org/licenses/by-nc/4.0/), which permits any noncommercial use, distribution, and reproduction in any medium, provided you give appropriate credit to the original author(s) and the source, provide a link to the Creative Commons license, and indicate if changes were made.

\section{References}

1. Scheller EL, Doucette CR, Learman BS, Cawthorn WP, Khandaker S, Schell B, Wu B, Ding SY, Bredella MA, Fazeli PK, Khoury B, Jepsen KJ, Pilch PF, Klibanski A, Rosen CJ, MacDougald OA (2015) Regionspecific variation in the properties of skeletal adipocytes reveals regulated and constitutive marrow adipose tissues. Nat Commun 6:7808

2. Devlin MJ, Rosen CJ (2015) The bone-fat interface: basic and clinical implications of marrow adiposity. Lancet Diabetes Endocrinol 3:141-147

3. Baum T, Cordes C, Dieckmeyer M, Ruschke S, Franz D, Hauner H, Kirschke JS, Karampinos DC (2016) MR-based assessment of body fat distribution and characteristics. Eur J Radiol 85:15121518
4. Baum T, Yap SP, Dieckmeyer M, Ruschke S, Eggers H, Kooijman H, Rummeny EJ, Bauer JS, Karampinos DC (2015) Assessment of whole spine vertebral bone marrow fat using chemical shift-encoding based water-fat MRI. J Magn Reson Imaging 42:1018-1023

5. Schwartz AV (2015) Marrow fat and bone: review of clinical findings. Front Endocrinol (Lausanne) 6:40

6. Sambrook P, Cooper C (2006) Osteoporosis. Lancet 367:2010 2018

7. Justesen J, Stenderup K, Ebbesen EN, Mosekilde L, Steiniche T, Kassem M (2001) Adipocyte tissue volume in bone marrow is increased with aging and in patients with osteoporosis. Biogerontology 2:165-171

8. Baum T, Yap SP, Karampinos DC, Nardo L, Kuo D, Burghardt AJ, Masharani UB, Schwartz AV, Li X, Link TM (2012) Does vertebral bone marrow fat content correlate with abdominal adipose tissue, lumbar spine bone mineral density, and blood biomarkers in women with type 2 diabetes mellitus? J Magn Reson Imaging 35:117-124

9. Heymsfield SB, Smith R, Aulet M, Bensen B, Lichtman S, Wang J, Pierson RN Jr (1990) Appendicular skeletal muscle mass: measurement by dual-photon absorptiometry. Am J Clin Nutr 52:214-218

10. Bolotin HH, Sievanen H, Grashuis JL, Kuiper JW, Jarvinen TL (2001) Inaccuracies inherent in patient-specific dual-energy X-ray absorptiometry bone mineral density measurements: comprehensive phantom-based evaluation. J Bone Miner Res 16:417-426

11. Antonacci MD, Hanson DS, Heggeness MH (1996) Pitfalls in the measurement of bone mineral density by dual energy x-ray absorptiometry. Spine (Phila Pa 1976) 21:87-91

12. Schuit SC, van der Klift M, Weel AE, de Laet CE, Burger H, Seeman E, Hofman A, Uitterlinden AG, van Leeuwen JP, Pols HA (2004) Fracture incidence and association with bone mineral density in elderly men and women: the Rotterdam Study. Bone 34: 195-202

13. Karampinos DC, Ruschke S, Dieckmeyer M, Diefenbach M, Franz D, Gersing AS, Krug R, Baum T (2018) Quantitative MRI and spectroscopy of bone marrow. J Magn Reson Imaging 47:332-353

14. Cordes C, Baum T, Dieckmeyer M, Ruschke S, Diefenbach MN, Hauner H, Kirschke JS, Karampinos DC (2016) MR-based assessment of bone marrow fat in osteoporosis, diabetes, and obesity. Front Endocrinol (Lausanne) 7:74

15. Ruschke S, Pokorney A, Baum T, Eggers H, Miller JH, Hu HH, Karampinos DC (2017) Measurement of vertebral bone marrow proton density fat fraction in children using quantitative water-fat MRI. MAGMA 30:449-460

16. Baum T, Rohrmeier A, Syvari J, Diefenbach MN, Franz D, Dieckmeyer M, Scharr A, Hauner H, Ruschke S, Kirschke JS, Karampinos DC (2018) Anatomical variation of age-related changes in vertebral bone marrow composition using chemical shift encoding-based water-fat magnetic resonance imaging. Front Endocrinol (Lausanne) 9:141

17. Dieckmeyer M, Ruschke S, Cordes C, Yap SP, Kooijman H, Hauner H, Rummeny EJ, Bauer JS, Baum T, Karampinos DC (2015) The need for T(2) correction on MRS-based vertebral bone marrow fat quantification: implications for bone marrow fat fraction age dependence. NMR Biomed 28:432-439

18. Shah LM, Hanrahan CJ (2011) MRI of spinal bone marrow: part I, techniques and normal age-related appearances. AJR Am J Roentgenol 197:1298-1308

19. Silva JR Jr, Hayashi D, Yonenaga T, Fukuda K, Genant HK, Lin C, Rahmouni A, Guermazi A (2013) MRI of bone marrow abnormalities in hematological malignancies. Diagn Interv Radiol 19:393-399

20. Schlaeger S, Inhuber S, Rohrmeier A, Dieckmeyer M, Freitag F, Klupp E, Weidlich D, Feuerriegel G, Kreuzpointner F, Schwirtz A, Rummeny EJ, Zimmer C, Kirschke JS, Karampinos DC, Baum T (2018) Association of paraspinal muscle water-fat MRI-based measurements with isometric strength measurements. Eur Radiol 
21. Mookiah MRK, Subburaj K, Mei K, Kopp FK, Kaesmacher J, Jungmann PM, Foehr P, Noel PB, Kirschke JS, Baum T (2018) Multidetector computed tomography imaging: effect of sparse sampling and iterative reconstruction on trabecular bone microstructure. J Comput Assist Tomogr 42:441-447

22. Haralick KSRM, Disntein I (1973) Textural features for image classification. IEEE Trans Syst Man Cybern SMC-3:610-621

23. Karahaliou AN, Boniatis IS, Skiadopoulos SG, Sakellaropoulos FN, Arikidis NS, Likaki EA, Panayiotakis GS, Costaridou LI (2008) Breast cancer diagnosis: analyzing texture of tissue surrounding microcalcifications. IEEE Trans Inf Technol Biomed 12: $731-738$

24. Link TM, Heilmeier U (2016) Bone quality-beyond bone mineral density. Semin Musculoskelet Radiol 20:269-278

25. Freedman DaPD (1981) On the histogram as a density estimator: L 2 theory. Probab Theory Relat Fields 57:453-476

26. Scott DW (1979) On optimal and data-based histograms. Biometrika 66:605-610

27. Sturges HA (1926) The choice of a class interval. J Am Stat Assoc 21:65-66

28. Tixier F, Le Rest CC, Hatt M, Albarghach N, Pradier O, Metges JP, Corcos L, Visvikis D (2011) Intratumor heterogeneity characterized by textural features on baseline 18F-FDG PET images predicts response to concomitant radiochemotherapy in esophageal cancer. J Nucl Med 52:369-378

29. Hanley JA, McNeil BJ (1982) The meaning and use of the area under a receiver operating characteristic (ROC) curve. Radiology 143:29-36

30. Kim SY, Kim EK, Moon HJ, Yoon JH, Kwak JY (2015) Application of texture analysis in the differential diagnosis of benign and malignant thyroid nodules: comparison with gray-scale ultrasound and elastography. AJR Am J Roentgenol 205:W343-W351

31. Brynolfsson P, Nilsson D, Torheim T, Asklund T, Karlsson CT, Trygg J, Nyholm T, Garpebring A (2017) Haralick texture features from apparent diffusion coefficient (ADC) MRI images depend on imaging and pre-processing parameters. Sci Rep 7:4041
32. Zayed N, Elnemr HA (2015) Statistical analysis of Haralick texture features to discriminate lung abnormalities. Int J Biomed Imaging 2015:267807

33. Ortiz-Ramon R, Larroza A, Ruiz-Espana S, Arana E, Moratal D (2018) Classifying brain metastases by their primary site of origin using a radiomics approach based on texture analysis: a feasibility study. Eur Radiol 28:4514-4523

34. Lisson CS, Lisson CG, Flosdorf K, Mayer-Steinacker R, Schultheiss M, von Baer A, Barth TFE, Beer AJ, Baumhauer M, Meier R, Beer M, Schmidt SA (2018) Diagnostic value of MRIbased 3D texture analysis for tissue characterisation and discrimination of low-grade chondrosarcoma from enchondroma: a pilot study. Eur Radiol 28:468-477

35. Meng Y, Zhang C, Zou S, Zhao X, Xu K, Zhang H, Zhou C (2018) MRI texture analysis in predicting treatment response to neoadjuvant chemoradiotherapy in rectal cancer. Oncotarget 9:1199912008

36. Mannil M, Burgstaller JM, Thanabalasingam A, Winklhofer S, Betz M, Held U, Guggenberger R (2018) Texture analysis of paraspinal musculature in MRI of the lumbar spine: analysis of the lumbar stenosis outcome study (LSOS) data. Skelet Radiol 47:947-954

37. Kricun ME (1985) Red-yellow marrow conversion: its effect on the location of some solitary bone lesions. Skelet Radiol 14:10-19

38. Buie HR, Moore CP, Boyd SK (2008) Postpubertal architectural developmental patterns differ between the L3 vertebra and proximal tibia in three inbred strains of mice. J Bone Miner Res 23:20482059

39. Mayerhoefer ME, Szomolanyi P, Jirak D, Materka A, Trattnig S (2009) Effects of MRI acquisition parameter variations and protocol heterogeneity on the results of texture analysis and pattern discrimination: an application-oriented study. Med Phys 36:1236-1243

Publisher's note Springer Nature remains neutral with regard to jurisdictional claims in published maps and institutional affiliations. 